\title{
Real-Time Three-Dimensional Reconstruction of Intraoperative Liver Surface Based on Structured Light Technique
}

\author{
Long Chen ${ }^{1}$ and JingLin $\mathrm{Li}^{2 *}$ \\ ${ }^{1}$ School of Mechanical and Electrical Engineering, Soochow University, China \\ ${ }^{2}$ School of logistics, Beijing Wuzi University, China \\ *Corresponding author: JingLin Li, School of logistics, Beijing Wuzi University, Beijing 101149, China. \\ To Cite This Article: JingLin Li, Real-Time Three-Dimensional Reconstruction of Intraoperative Liver Surface Based on Structured Light Technique. \\ Am J Biomed Sci \& Res. 2020 - 7(2). AJBSR.MS.ID.001132. DOI: 10.34297/AJBSR.2020.07.001132.
}

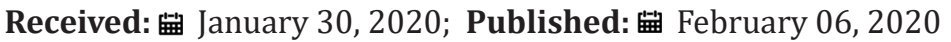

\section{Mini Review}

The incidence of liver tumor is increasing year by year, and liver tumor resection is a routine operation for liver lesions. Liver tumor resection is more difficult than general surgery, mainly reflected in the liver itself is easy to deform, vascular density, complex structure and other aspects [1]. If the three-dimensional information of the liver surface can be obtained in real time during the operation, it will be helpful for doctors to observe the cutting status of the liver surface in real time and avoid the possibility of being cut by mistake to a large extent. Clinical studies have shown that failure to accurately locate the focal point of the liver during surgery, to obtain real-time three-dimensional information on the liver surface and to plan the surgical path are the most important reasons for the failure of such surgery [2].

Traditional liver tumor resection surgery mainly relies on the doctor's own experience, hand feel, visual observation to determine the location of the tumor, to obtain real-time information of the liver cutting surface. In the field of computer-assisted surgery, there is no good way to provide real-time three-dimensional reconstruction of the liver surface during surgery. It also fails to provide color and high definition on the surface of the doctor's liver so that the texture of the liver surface can be well recognized.

In order to solve these problems, surgical navigation technology (IGS), which is represented by real-time three-dimensional reconstruction of intraoperative liver surface, has gradually entered scientists' research field of vision [3]. Real-time three-dimensional reconstruction of intraoperative liver surface based on structured light is one of the hot topics in the field of medicine and engineering. It is a combination of medical, optical, instrument and information technology, aiming at using structural light for real-time three- dimensional reconstruction of liver surface during surgery [4]. In this way, the accuracy and timeliness of the three-dimensional reconstruction of the organ surface during the operation can be further improved [5]. Especially in operations with significant soft-tissue manipulation or deformation, such as tumor resection of the liver, where the deformation is larger, the incision is deeper, and the operation is more extensive. At this point, real-time threedimensional liver reconstruction based on structural light can give full play to the advantages of good real-time performance and high accuracy, thus greatly reducing the risk of surgery. Compared with the traditional method of intraoperative liver surface information collection, the real-time intraoperative liver surface information collection method and system based on structural light scanning has the advantages of no contact, no radiation, anti-interference and strong adaptability, which is suitable for various operating environments.

Starting from the 21st century, the 3D reconstruction technology based on structural light has been relatively mature in industrial and architectural industries. In the medical industry, the technology of structural light for $3 \mathrm{~d}$ reconstruction is not yet mature, and only a few technologies can be used for 3D reconstruction of endoscopic intestinal tract. Currently, there are few researches on the introduction of structural light to achieve real-time reconstruction of intraoperative liver surface. The United States, the United Kingdom and Canada are among the world's leading researchers in this field, and some research teams have made outstanding achievements in applying structural light technology to three-dimensional reconstruction of liver, lung, spine and other organs or human body parts. 
In 2012, the university of nuremberg in Germany proposed a new endoscope 3D scanning system based on monocular structured light, integrating the camera receiving the pattern and the pattern projection unit into the sensor. The sensor head is only $3.6 \mathrm{~mm}$ in diameter and $14 \mathrm{~mm}$ in length and is mounted on a flexible shaft [6]. Capture 3D video at a rate of 30 frames per second, typically generating about 5,000 3D points per frame. In 2015, imperial college London in the United Kingdom developed an endoscopic structured light system for intestinal examination, called the miniature structured light (SL) system [7]. It is mainly used for rapid recovery of tissue surface shape in minimally invasive surgery (MIS). The average accuracy of three-dimensional reconstruction of the intestinal inner surface has reached less than $1 \mathrm{~mm}$, and the accuracy has completely reached the clinical requirement of less than $2 \mathrm{~mm}$. At the 34 th IEEE EMBS international conference, the university of Strasbourg, France, presented a novel three-dimensional laparoscopic device based on structured light for minimally invasive surgery [8]. In October 2018, structured light was used by the university of Toronto in Canada for high-speed, high-density intraoperative 3D reconstruction, which was used for effective registration of MRI and CT images in the navigation of cranial and spinal surgery [9]. The experimental navigation system of optical topology imaging (OTI) was developed to obtain the 3D surface anatomy of the surgical cavity. The system is much faster than commercial reference systems and does not affect spatial accuracy.

At present, the research on 3D reconstruction technology of human organs has made a breakthrough to a certain extent. The 3D reconstruction effect of similar rigid bodies (such as spine and bone, etc.) has basically met the needs of doctors in the process of surgery. However, for soft tissue (such as liver, heart and lung, etc.), there has not been a breakthrough. For the existing 3D soft tissue reconstruction system, most of them can only stay in the experimental stage, failing to meet the clinical requirements. The three-dimensional reconstruction of the spine based on structured light technology has achieved good results, which can be combined with medical principles and introduced into the real-time threedimensional reconstruction of intraoperative liver.

The need to achieve better three-dimensional reconstruction of liver surface is also closely related to optics, information technology, human-computer interaction and other disciplines. A new, more suitable, more perfect and more humane scheme should be proposed from the perspective of both patients and doctors. Real-time acquisition of intraoperative liver surface information based on structured light is the basis of liver surgery navigation, so it requires high real-time and precision of three-dimensional reconstruction. At present, the liver surface information collection and real-time 3D reconstruction based on structured light still have some problems, such as poor real-time performance, low precision, and light shielding. With the integration of structured light technology into medical surgery and modern computer technology, three-dimensional reconstruction of liver surface based on structured light is possible. It will bring great breakthroughs to the medical robot industry and create great social benefits for human medical surgery [10].

\section{References}

1. Lavanya Yohanathan, Sean P Cleary (2019) Minimally Invasive Management of Secondary Liver Cancer. Surg Oncol Clin N Am 28(2): 229-241.

2. Clements, Logan W, Collins, Jarnagin WR, Miga MI, et al. (2017) Deformation correction for image-guided liver surgery: An intraoperative fidelity assessment. Surgery 162(3): 537-547.

3. Ahmed K. Image 2015 Guided Surgery. BJO 20(1): 1-4.

4. Sugawara M, Kiyomitsu K, Namae T, Toshiya Nakaguchi. (2016) An optical projection system with mirrors for laparoscopy. Artificial Life \& Robotics 22(1): 1-7.

5. Cash D M, Sinha T K, Chapman W C, Terawaki H, Dawant BM, et al. (2003) Incorporation of a laser range scanner into image-guided liver surgery: Surface acquisition, registration, and tracking. Med Phys 30(7): 1671

6. Schmalz C, Forster F, Schick A, E Angelopoulou (2012) An endoscopic 3D scanner based on structured light. Medical image analysis 16(5): 10631072.

7. Lin J, Clancy N T, Elson DS (2015) An endoscopic structured light system using multispectral detection. Int J Comput Assist Radiol Surg 10(12): 1941-1950.

8. Maurice X, Albitar C, Doignon C (2012) A structured light-based laparoscope with real-time organs' surface reconstruction for minimally invasive surgery. Conf Proc IEEE Eng Med Biol Soc 2012: 5769-5772.

9. Jakubovic R, Guha D, Gupta S, Lu M, Jivraj J, et al. (2018) High Speed, High Density Intraoperative 3D Optical Topographical Imaging with Efficient Registration to MRI and CT for Craniospinal Surgical Navigation. Sci Rep 8(1): 14894

10. Hu ZHZ, Guan QGQ Liu SLS, Chen SY, et al. (2009) Robust 3D Shape Reconstruction from a Single Image Based on Color Structured Light. International Conference on Artificial Intelligence \& Computational Intelligence. IEEE Computer Society. 